\title{
HYBRID GEOMETRIC CALIBRATION METHOD FOR MULTI-PLATFORM SPACEBORNE SAR IMAGE WITH SPARSE GCPS
}

\author{
Guannan.Lv ${ }^{1,2, *}$, Xinming.Tang ${ }^{2}$, Bo.Ai ${ }^{1}$, Tao.Li ${ }^{2}$, Qianfu.Chen ${ }^{2}$ \\ ${ }^{1}$ College of Geomatics, Shandong University of Science and Technology, Qingdao, China - lvgn@ foxmail.com, 4308954@qq.com \\ ${ }^{2}$ Satellite Surveying and Mapping Application Center, NASG, Beijing, China - tangxinming99@ qq.com, (lit, chenqf)@ sasmac.cn
}

Commission III, WG III/3

KEY WORDS: SAR image, geometric calibration, R-D model, multi-platform, TerraSAR-X, TanDEM-X, Gaofen-3

\begin{abstract}
:
Geometric calibration is able to provide high-accuracy geometric coordinates of spaceborne SAR image through accurate geometric parameters in the Range-Doppler model by ground control points (GCPs). However, it is very difficult to obtain GCPs that covering large-scale areas, especially in the mountainous regions. In addition, the traditional calibration method is only used for single platform SAR images and can't support the hybrid geometric calibration for multi-platform images. To solve the above problems, a hybrid geometric calibration method for multi-platform spaceborne SAR images with sparse GCPs is proposed in this paper. First, we calibrate the master image that contains GCPs. Secondly, the point tracking algorithm is used to obtain the tie points (TPs) between the master and slave images. Finally, we calibrate the slave images using TPs as the GCPs. We take the Beijing-TianjinHebei region as an example to study SAR image hybrid geometric calibration method using 3 TerraSAR-X images, 3 TanDEM-X images and 5 GF-3 images covering more than 235 kilometers in the north-south direction. Geometric calibration of all images is completed using only 5 GCPs. The GPS data extracted from GNSS receiver are used to assess the plane accuracy after calibration. The results after geometric calibration with sparse GCPs show that the geometric positioning accuracy is 3m for TSX/TDX images and $7.5 \mathrm{~m}$ for GF-3 images.
\end{abstract}

\section{INTRODUCTION}

Geometric calibration technology is the key to achieve highaccuracy positioning of SAR images, and is also one of the main ways to improve the accuracy of geometric positioning without GCPs. The high-accuracy geometry calibration of TerraSAR satellite is accomplished using corner reflector, which can realize the accuracy of $\mathrm{cm}$-level positioning (Eineder et al., 2011a; Cong et al., 2012a; Zhou et al., 2014a). The TerraSAR-X is the civilian satellite with the highest resolution currently in orbit. Schwerdt et al. (2007) and Schwerdt et al. (2010a) summarized the geometric calibration accuracy of TerraSAR-X, the azimuth direction is $0.5 \mathrm{~m}$, and the range distance is $0.3 \mathrm{~m}$. After the launch of GF-3, Zhang (2017a) and Ding et al. (2017a) summarized its positioning error and verified that the accuracy of the geometric positioning was up to about $3 \mathrm{~m}$ after the geometric calibration.

We presents a hybrid geometric calibration method for multiplatform spaceborne SAR image with sparse GCPs. First, we calibrate the master image that contains GCPs. Secondly, the TPs tracking algorithm is used to obtain the TPs between the master and slave images. Finally, we calibrate the slave images using TPs as the GCPs. The method breaks through the limitation of the traditional geometric calibration method, which can only calibrate the single platform SAR images, and can simultaneously realize the geometric calibration of the multiplatform spaceborne SAR image. We take the Beijing-TianjinHebei region as an example to study SAR image hybrid geometric calibration method using 3 TerraSAR-X images, 3 TanDEM-X images and 5 GF- 3 images covering more than 235 kilometers in the north-south direction. The GPS data extracted from GNSS receiver are used to assess the plane accuracy.

\section{METHOD}

This method mainly reflects the idea of transitive geometric calibration, the process shown in Figure 1. First, the SAR images (main images) containing the measured GCPs is selected. Based on the R-D model, the nonlinear least square principle is used to refine the model parameters and determine the calibration parameters to complete the geometric calibration of the main image. Then, the geometric parameters are used to compute the adjacency images (slave images) with overlapped areas of main images. The TPs tracking algorithm is used to extract the TPs between the main and the slave images, and the coordinates of the TPs are computed by using the main image and SRTM. Finally, the geometric calibration of the slave images is completed based on the TPs.

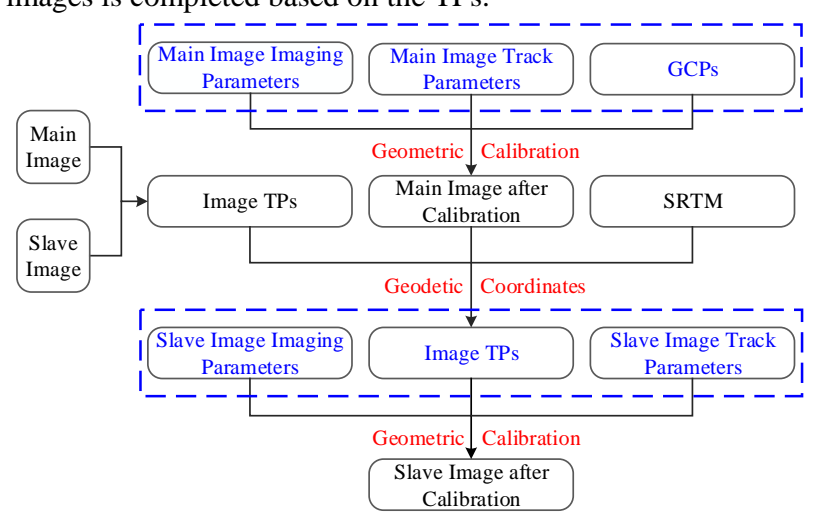

Figure 1. Flowchart of hybrid geometric calibration 


\subsection{Geometric Calibration of Spaceborne SAR Image}

As the mainstream positioning model of SAR geometry processing, R-D model is a rigorous model that accords with the SAR imaging mechanism. It describes the geometric relationship between satellite sensor and ground object in the geocentric coordinate system (Yang, 2016b). The R-D model is a set of nonlinear equations consisting of the Range equation (1), the Doppler equation (2) and the Earth-ellipsoid equation (3) (Curlander et al., 1991).

$$
\begin{gathered}
R=\left|\mathbf{R}_{s c}-\mathbf{R}_{t c}\right| \\
f_{d}=-\frac{2}{\lambda} \frac{\left(\mathbf{R}_{s c}-\mathbf{R}_{t c}\right) \cdot \mathbf{V}_{s c}}{R} \\
\frac{X_{t}^{2}+Y_{t}^{2}}{\left(R_{e}+H_{t}\right)^{2}}+\frac{Z_{t}^{2}}{R_{p}^{2}}=1
\end{gathered}
$$

where $R=$ distance between the satellite sensor and the ground object

$\mathbf{R}_{s c}=\left[\begin{array}{lll}X_{s} & Y_{s} & Z_{s}\end{array}\right]^{\mathrm{T}}=$ position vector of the satellite

sensor

$\mathbf{R}_{t c}=\left[\begin{array}{lll}X_{t} & Y_{t} & Z_{t}\end{array}\right]^{\mathrm{T}}=$ position vector of the ground

object

$f_{d}=$ Doppler frequency

$\lambda=$ radar wavelength

$\mathbf{V}_{s c}=\left[\begin{array}{lll}V_{x} & V_{y} & V_{z}\end{array}\right]^{\mathrm{T}}=$ velocity vector of the satellite

sensor

$R_{e}=$ major semi-axes of the earth reference ellipsoid

$R_{p}=$ minor semi-axes of the earth reference ellipsoid

$H_{t}=$ elevation of the ground object

The position and velocity vector of the satellite sensor can be described as a function of time by cubic polynomials, as shown in Equation (4).

$$
\left\{\begin{array} { l } 
{ X _ { s } = a _ { 0 } + a _ { 1 } t + a _ { 2 } t ^ { 2 } + a _ { 3 } t ^ { 3 } } \\
{ Y _ { s } = b _ { 0 } + b _ { 1 } t + b _ { 2 } t ^ { 2 } + b _ { 3 } t ^ { 3 } } \\
{ Z _ { s } = c _ { 0 } + c _ { 1 } t + c _ { 2 } t ^ { 2 } + c _ { 3 } t ^ { 3 } }
\end{array} \quad \left\{\begin{array}{l}
V_{x}=a_{1}+2 a_{2} t+3 a_{3} t^{2} \\
V_{y}=b_{1}+2 b_{2} t+3 b_{3} t^{2} \\
V_{z}=c_{1}+2 c_{2} t+3 c_{3} t^{2}
\end{array}\right.\right.
$$

According to the R-D model, the necessary variables of geometric positioning include the oblique distance $R$, the position vector of the satellite sensor $\left[\begin{array}{lll}X_{s} & Y_{s} & Z_{s}\end{array}\right]^{\mathrm{T}}$, the velocity vector of the satellite sensor $\left[\begin{array}{lll}V_{x} & V_{y} & V_{z}\end{array}\right]^{\mathrm{T}}$, Doppler frequency $f_{d}$, radar wavelength $\lambda$, and ellipsoid parameters $R_{e} / R_{p}$. Among them, the radar wavelength is known, and can be obtained according to the equation $\lambda=c / f$ ( $c$ is the speed of light, $f$ is the radar load frequency). The ellipsoid parameters are also known after the ellipsoid model is selected.

The slant distance $R$ is obtained by measuring the time difference between the sending and receiving of the radar pulse. The oblique distance error will cause the field of view of the target to move along the Doppler line, resulting in the positioning error. For slant range SAR images, the slant distance can be expressed as a linear function of the image column number:

$$
R=r_{0}+m_{j} \cdot j
$$

where $\quad r_{0}=$ near slant of image

$$
\begin{aligned}
& m_{j}=\text { pixel resolution of range direction } \\
& j=\text { image column number increasing from the near }
\end{aligned}
$$

to the far slant of image

It is generally considered that the resolution of the distance pixel provided in the header file is accurate. The previous experiments also found that the influence of the corrected distance pixel resolution on the final positioning result is negligible, so only the near slant error on the impact of the distance to be considered. It is known from the Equation (4) that the position and velocity state vector error of the satellite sensor is closely related to the azimuth time $t$. The azimuth time $t$ can be expressed as a linear function of the line number of the image:

$$
t=t_{0}+t_{1} \cdot i
$$

where $t_{0}=$ initial imaging time of the image

$t_{1}=$ azimuth time resolution

$i=$ line number of the image that gradually increases along the azimuth

The experiment shows that the influence of azimuth time resolution error on the final positioning result is also negligible, so we only consider the influence of initial imaging time error on azimuth positioning. In the imaging process of spaceborne SAR, we need to conduct yaw guidance to the satellite, so as to ensure that the Doppler value is near zero, and zero Doppler imaging is completed in the image focusing stage, so that all azimuth images are zero to the Doppler parameter. Therefore, the Doppler parameters in this paper no longer be extra calibration (Cao et al., 1999a). In summary, this paper only considers the influence of the measurement errors on the geometric positioning accuracy of the near range and the initial imaging time, so the parameters vector of the geometric calibration is $\boldsymbol{\Sigma}=\left[\begin{array}{ll}r_{0} & t_{0}\end{array}\right]^{\mathrm{T}}$. The conditional equation based on the distance equation (see Eq.(1)) is as follows:

$$
F_{1}(\boldsymbol{\Sigma})=\left|\mathbf{R}_{s c}-\mathbf{R}_{c c}\right|-\left(r_{0}+m_{j} \cdot j\right)
$$

The conditional equation based on the Doppler frequency equation (see Eq.(2)) is as follows:

$$
F_{2}(\Sigma)=-\frac{2}{\lambda} \cdot \frac{\left(\mathbf{R}_{s c}-\mathbf{R}_{t c}\right) \cdot \mathbf{V}_{s c}}{R}
$$

Since there are no imaging parameters involved in the Earth ellipsoid equation (see Eq.(3)), it is not necessary to establish conditional equations. The nonlinear least squares estimation of the model parameters can be carried out by a number of calibration points by the conditional equation. The calibration parameters vector $\boldsymbol{\Sigma}$ is used as an unknown observation value, and the error equation is obtained by linearizing the observation value derivation:

$$
\left\{\begin{array}{l}
V_{1}=\left(F_{1}\right)-F_{1}+\frac{\partial F_{1}}{\partial r_{0}} \Delta r_{0}+\frac{\partial F_{1}}{\partial t_{0}} \Delta t_{0} \\
V_{2}=\left(F_{2}\right)-F_{2}+\frac{\partial F_{2}}{\partial r_{0}} \Delta r_{0}+\frac{\partial F_{2}}{\partial t_{0}} \Delta t_{0}
\end{array}\right.
$$

In Equation (9), the observational error $\left(F_{1}\right)$ and $\left(F_{2}\right)$ can be calculated by the approximation of the approximate values of 
each undetermined parameter (7) and (8). The true value of the observation is $F_{1}=F_{2}=0$. When a number of calibration GCPs are used to optimize the parameters of the model, the error equation can be written into a matrix form:

$$
\mathbf{V}=\mathbf{B X}-\mathbf{L}
$$

where

$$
\mathbf{V}=\left[\begin{array}{l}
V_{1} \\
V_{2}
\end{array}\right], \quad \mathbf{B}=\left[\begin{array}{ll}
\frac{\partial F_{1}}{\partial r_{0}} & \frac{\partial F_{1}}{\partial t_{0}} \\
\frac{\partial F_{2}}{\partial r_{0}} & \frac{\partial F_{2}}{\partial t_{0}}
\end{array}\right], \quad \mathbf{X}=\left[\begin{array}{l}
\Delta r_{0} \\
\Delta t_{0}
\end{array}\right], \mathbf{L}=\left[\begin{array}{l}
F_{1}-\left(F_{1}\right) \\
F_{2}-\left(F_{2}\right)
\end{array}\right]
$$

Given the initial value of the parameter $X_{0}$, and the normal equation $\mathbf{B}^{\mathrm{T}} \mathbf{B X}-\mathbf{B}^{\mathrm{T}} \mathbf{L}=0$ is constructed. The solution of the normal equation and the unknown correction amount $\mathbf{X}=\left(\mathbf{B}^{\mathrm{T}} \mathbf{B}\right)^{-1} \mathbf{B}^{\mathrm{T}} \mathbf{L}$ can be obtained, and the first correction value of the calibration parameters can be obtained:

$$
X_{0}+X=\left[\begin{array}{ll}
r_{0}+\Delta r & t_{0}+\Delta t
\end{array}\right]^{\mathrm{T}}
$$

The process of solving the calibration parameters is an iterative approach. The basic idea is as follows: first, the initial value of calibration parameters $r_{0}$ and $t_{0}$ is given, and the error equation is constructed according to the initial value. The corrected value of calibration parameters is solved by the normal equation, and the first approaching value of calibration parameters is obtained. The approaching value is taken as the initial value of the next iteration. After several iterations, the calibration parameters are gradually stable. When the correction value is less than the specified threshold $\left(\varepsilon=10^{-6}\right)$, the convergence of iteration is achieved. At this time, the cumulative result of correction value is the final calibration parameters correction value.

\subsection{TPs Tracking Algorithm}

Geometric calibration of slave image depends on the calibration result of main image, it is necessary to find a relationship between main and slave images before correcting the image. In this paper, we establish this connection by finding the TPs between overlapping images. The TPs reflect the mapping relationship between the main image points and the slave image points. The TPs plays a controlling role in the calibration of the slave image, but it has two main advantages compared to the GCP. First, the TPs tracking algorithm can achieve the accuracy of sub-pixel matching, which can reduce the error of manual operation. Secondly, the TPs tracking algorithm can be automated through the program, which can reduce the cost of manual measurement.

The resolution of SAR images is different in different platforms. In order to ensure the matching accuracy of TPs, we need to pre-process the images to make the resolution of images consistent, and then use TPs tracking algorithm to locate and match the TPs. First, according to the principle of uniform distribution, several points with strong backscattering coefficients are selected as the initial TPs on the main image. Secondly, the TPs tracking algorithm is used to obtain the TPs coordinate coordinates in the slave image. Finally, all TPs are polynomial fitting, and the mismatched TPs are eliminated. Based on the relevant algorithms of SAR image registration, the core algorithm of TPs tracking algorithm includes two parts: the TPs coarse positioning and the fine matching.
2.2.1 TPs Coarse Positioning: The purpose of the TPs coarse positioning is to provide the initial offset and the search window for the fine matching algorithm. The coarse positioning is based on the R-D model and the main and slave image track parameters for calculation. The geometric positioning accuracy is within 10 pixels.

The core of the coarse positioning algorithm consists of two aspects, namely the direct geometric positioning algorithm and the indirect geometric positioning algorithm (Chen, 2004b). Among them, the former realizes the conversion between the central point $M(i, j)$ of the main image and the ground point $P(x, y, z)$, and the latter realizes the transformation from the ground point $P(x, y, z)$ to the matching point $S\left(i^{\prime}, j^{\prime}\right)$ corresponding to the slave image. The two conversion processes are based on the same precondition that each image point of SAR image corresponds to a single imaging time and a specific range value (see Eq.(5), (6)). The initial offset $\Delta$ of the TPs obtained through the above process can be obtained by Equation (12).

$$
\Delta=\left\{\begin{array}{c}
\Delta i=i^{\prime}-i \\
\Delta j=j^{\prime}-j
\end{array}\right.
$$

2.2.2 TPs Fine Positioning: Normalized Cross Correlation (NCC) matching method can match the gray information in the matching window of two images, which has a certain anti-noise ability. The NCC equation is as follows (Guillaume et al., 2004a):

$$
\rho=\frac{1}{n} \frac{\sum_{i=1}^{n}\left(m_{i}-\bar{m}\right)\left(s_{i}-\bar{s}\right)}{\sigma_{m} \sigma_{s}}
$$

where $\quad m_{i}=$ pixel value in the main image

$s_{i}=$ pixel value in the slave image

$\bar{m}=$ pixel average value in the main image

$\bar{s}=$ pixel average value in the slave image

$\sigma_{m}=$ pixel standard deviation in the main image

$\sigma_{s}=$ pixel standard deviation in the slave image

$n=$ number of pixel

The TPs matching will be refined on the basis of the initial offset to achieve sub-pixel-level matching accuracy. First of all, according to the offset and search window obtained by coarse positioning, we choose a window with $L \times L$ pixels from the initial TPs of the main image and the slave image, and calculate the pixels of each pixel in the window according to Equation (13), and get a window with $L \times L$ NCC values. Then, the NCC values in the window are surface-fitted to obtain the function expression of the NCC surface:

$$
Z=f(x, y)
$$

where $\quad(x, y)=$ coordination for image point

$$
Z=\text { NCC value of image point }
$$

Finally, according to the NCC surface function expression obtained by fitting, interpolating the NCC value with sub-pixel spacing, the maximum NCC value corresponds to the exact positioning of the TPs. 
2.2.3 Mismatch Point Elimination: In the fine matching stage, we use the coarse positioning results of the TPs with the global residual points of the TPs to eliminate the obvious mismatching points. However, due to the different radar band, imaging angle and scattering characteristics of multi-platform SAR images, there are still some mismatching points in the result of TPs tracking algorithm. The geometrical distortion of the adjacent image of SAR is small, which is usually expressed as translation, stretching and minimal rotation. The distortion characteristic similar to the rigid deformation can be modified by a polynomial combined with a partial two term. The more commonly used fitting polynomial can be expressed as Equation (15) (Hanssen et al., 2001a):

$$
\left\{\begin{array}{l}
a_{0}+a_{1} x_{m}+a_{2} y_{m}+a_{3} x_{m} y_{m}=x_{s} \\
b_{0}+b_{1} x_{m}+b_{2} y_{m}+b_{3} x_{m} y_{m}=y_{s}
\end{array}\right.
$$

where $\left(x_{m}, y_{m}\right)=$ point coordinates of the TPs in the main image

$\left(x_{s}, y_{s}\right)=$ point coordinates of the TPs in the slave

image

$$
a_{i}, b_{i}=\text { polynomial fitting coefficient, } i=0,1,2,3
$$

Mismatch point elimination is divided into two steps: First, according to the TPs, we calculate the parameters of the first polynomial model and calculate the residuals. When the residual is greater than 1 pixels, we reject the largest error point and recalculate the parameters and residuals of the polynomial model until the residual is less than 1 pixels. Then, the mean square deviation $\sigma_{x}$ and $\sigma_{y}$ in the horizontal and vertical direction are calculated, and the position deviation in the horizontal vertical direction is larger than the threshold of the threshold $T_{x}\left(T_{x}=3 \sigma_{x}\right)$ and $T_{\mathrm{y}}\left(T_{\mathrm{y}}=3 \sigma_{y}\right)$. It is worth noting that with the iterated iteration, the number of TPs will also gradually decrease. When the number of TPs is not enough to solve polynomial models, the TPs tracking algorithm is judged to be failure. At this time, we can expand the search window and increase the threshold setting to re-execute the TPs tracking algorithm until we generate enough number of points to meet sub-pixel accuracy requirements.

\subsection{TP's Geodetic Coordinate Acquisition Based on SRTM}

The TPs are all in pairs, and the same pair of points on the main and slave images should have the same geodetic coordinates. After the calibration of the main image is completed, the slave image needs to realize its own geometric calibration with the help of TPs. Therefore, the TPs also need geographic coordinates and elevation information. However, the TPs acquired by TPs tracking algorithm contain only the information of row and column in the image coordinate system. Therefore, we get the geodetic coordinates of the TPs by SRTM data iteratively, and the process as shown in Figure 2. First, we get the average elevation value of the study area and use it as the initial value of all TPs. Based on the image coordinates and elevation values $\left(H_{1}\right)$ of the main image TPs, we use the direct geometric positioning algorithm to get the geographic coordinates corresponding to the main image TPs. Then, we get the elevation value $\left(\mathrm{H}_{2}\right)$ of the geographic coordinate in the SRTM data to determine whether the two elevation difference is less than the set threshold $\left(\varepsilon=10^{-6}\right)$. If value does not meet the requirement, we will correct the elevation value and perform the next iteration. Finally, the image coordinates of the geodetic coordinates of the main image TPs and the slave image TPs are synthesized, and the complete coordinate information of the slave image TPs is obtained.

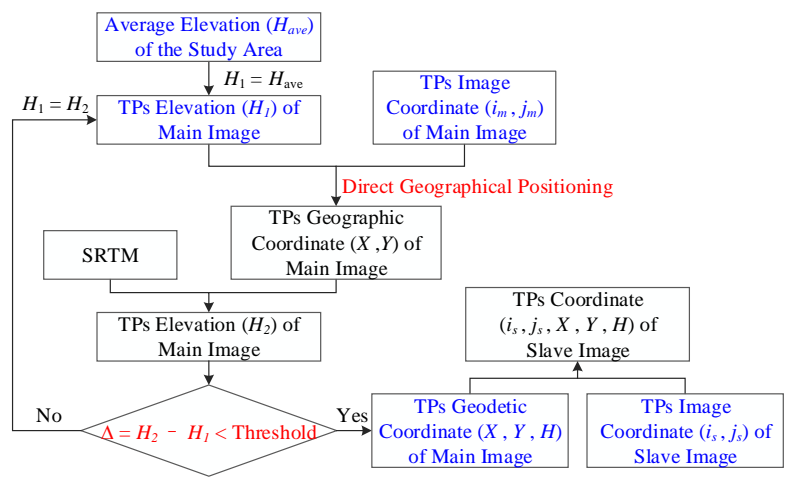

Figure 2. Flowchart of obtaining TP's geodetic coordinate based on SRTM DEM

\section{EXPERIMENTAL AND ANALYSIS}

\subsection{Experimental data}

Experimental data center area located at Beijing, latitude and longitude is $116^{\circ} 28^{\prime} \mathrm{E}$ and $39^{\circ} 55^{\prime} \mathrm{N}$, north-south distribution is $235 \mathrm{~km}$, both plain and mountainous area. The altitude is $16 \sim 1588 \mathrm{~m}$, its geographical position is shown in Figure 3. First, 196 alternative points were selected for SAR images based on optical images, which were mainly distributed in the intersection of the suitable width and easy identification of SAR images. Then, it adjusts and confirms the location of alternative points in the field, and abandoning some alternative points of some measurement difficulties according to the actual situation, such as larger road traffic and nearby tall buildings shelter.

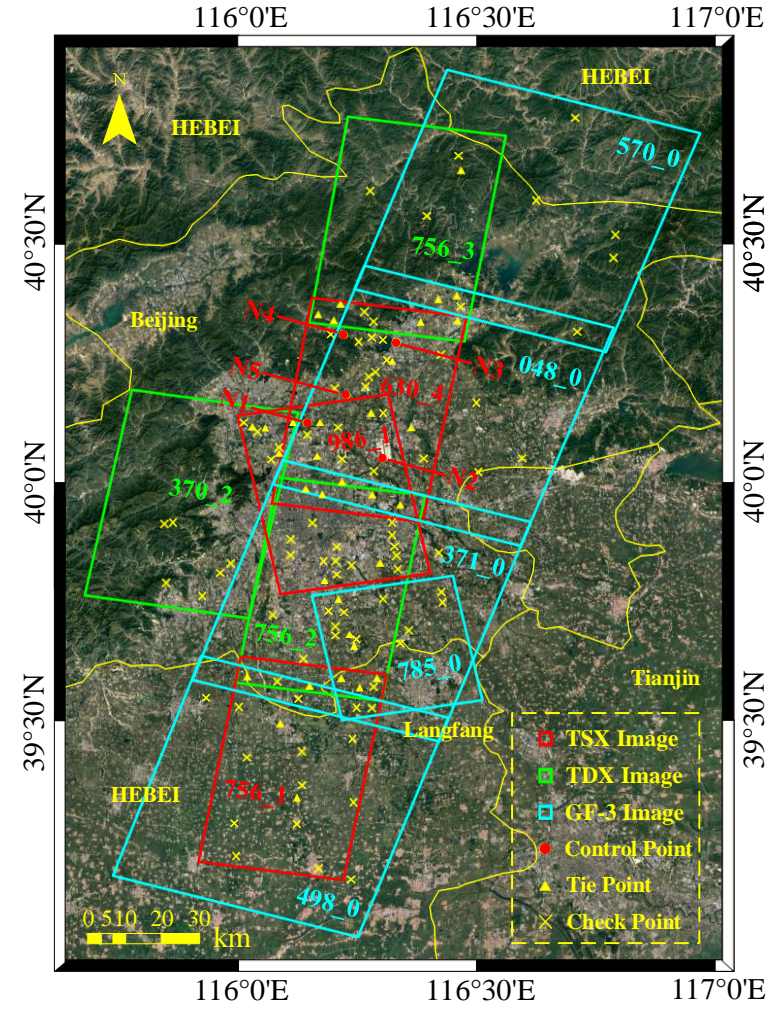

Figure 3. Geographical position and point's distribution of study region 
Then 87 GPS alternatives are screened out. Finally, the internal industry collate all the GPS points measured and pricked the GPS points on the SAR image. In the measured GPS point, 5 GCPs and 82 check points (CPs) are selected. In addition, the TPs tracking algorithm is used to extract 34 image TPs, and the detailed point distribution is shown in Figure 3.
Experimental data include 3 TSX images, 3 TDX images and 5 GF-3 images (time: 20131120-20160816). We chose SRTM (resolution: 90m) as the external DEM data. The basic parameters of the experimental image are shown in Table 1.

\begin{tabular}{|c|c|c|c|c|c|c|c|c|}
\hline Image ID & Platform & Time & Orbit Direction & $\begin{array}{c}\text { Average } \\
\text { Elevation/m }\end{array}$ & $\begin{array}{c}\text { Resolution of } \\
\text { Azimuth/m }\end{array}$ & $\begin{array}{c}\text { Resolution of } \\
\text { Range/m }\end{array}$ & Near Time/ms & Near Range/m \\
\hline $630 \_4$ & TSX & 20140316 & Descending & 221.598 & 2.00 & 1.36 & 80266935.211 & 690732.087 \\
\hline 986_1 & TSX & 20151119 & Ascending & 31.420 & 1.85 & 0.91 & 36266414.462 & 595457.579 \\
\hline $756 \_3$ & TDX & 20140316 & Descending & 488.793 & 2.00 & 1.36 & 80259935.119 & 690208.271 \\
\hline 370_2 & TDX & 20131120 & Descending & 282.103 & 1.97 & 1.36 & 80787910.594 & 597318.189 \\
\hline 785_0 & GF-3 & 20160908 & Ascending & 21.529 & 5.29 & 2.25 & 36515935.223 & 903671.719 \\
\hline 570_0 & GF-3 & 20160816 & Descending & 489.643 & 3.14 & 2.25 & 83209554.134 & 919669.644 \\
\hline 048_0 & GF-3 & 20160816 & Descending & 38.271 & 3.14 & 2.25 & 83217764.984 & 920101.346 \\
\hline 371_0 & GF-3 & 20160816 & Descending & 32.089 & 3.14 & 2.25 & 83225975.835 & 919977.681 \\
\hline
\end{tabular}

Table 1. Basic parameters of study images

\subsection{Results}

The geometric accuracy of the experimental image before calibration is evaluated by the indirect geometric positioning algorithm. First, the artificial prick point is carried out, and the GPS points are transferred to the image to obtain the image point coordinates $P_{1}$. Then, using the geodetic coordinates of the GPS points, the image point coordinates $P_{2}$ are calculated by using the indirect geometric positioning algorithm. Finally, $P_{2}$ and $P_{1}$ are subtracted to get the geometric positioning error $\Delta$ of the points, and the error of all points in azimuth and range direction is counted respectively (RMS, see Eq.(16)), so as to evaluate the geometric positioning accuracy of the image. The geometric accuracy evaluation before the calibration of the 11 experimental images with $82 \mathrm{CPs}$ is shown in Table 2.

$$
R M S=\sqrt{\frac{1}{n} \sum_{i=1}^{n} \Delta_{i}^{2}}
$$

\begin{tabular}{|c|c|c|c|c|c|c|c|c|c|}
\hline \multirow{2}{*}{ Image ID } & \multirow{2}{*}{$\begin{array}{l}\text { Number } \\
\text { of } \mathrm{CPs}\end{array}$} & \multicolumn{3}{|c|}{ Accuracy Evaluation Before Calibration } & \multirow{2}{*}{ Image ID } & \multirow{2}{*}{$\begin{array}{l}\text { Number } \\
\text { of } \mathrm{CPs}\end{array}$} & \multicolumn{3}{|c|}{ Accuracy Evaluation After Calibration } \\
\hline & & Range/m & Azimuth/m & Plane/m & & & Range/m & Azimuth $/ \mathrm{m}$ & Plane/m \\
\hline 630_4 & 11 & 3.967 & 2.686 & 4.791 & 785_0 & 7 & 26.172 & 6.173 & 26.890 \\
\hline 756_1 & 6 & 6.899 & 1.693 & 7.104 & 570_0 & 5 & 19.931 & 114.569 & 116.290 \\
\hline 986_1 & 7 & 20.400 & 381.378 & 381.923 & 048_0 & 8 & 20.969 & 113.488 & 115.409 \\
\hline 756_3 & 5 & 71.151 & 464.520 & 469.938 & 371_0 & 11 & 24.561 & 109.149 & 111.878 \\
\hline $756 \_2$ & 9 & 69.840 & 463.826 & 469.055 & 498_0 & 6 & 22.997 & 106.984 & 109.428 \\
\hline 370_2 & 7 & 5.705 & 2.240 & 6.129 & & & & & \\
\hline
\end{tabular}

$$
\begin{aligned}
& \text { where } \quad n=\text { the number of the GPS points } \\
& \Delta_{i}=\text { geometric positioning error of the } i \text {-th GPS point, } \\
& i=1,2, \cdots, n
\end{aligned}
$$

Table 2. Accuracy evaluation of study images before the geometric calibration

Table 1 and Table 2 shows that there are great differences in the geometric positioning accuracy of data products under different platforms. For example, the geometric positioning accuracy of 630_4 TSX image is $4.791 \mathrm{~m}$, while the geometric positioning accuracy of 570_0 GF-3 image is $116.290 \mathrm{~m}$. In addition, the geometric positioning accuracy of data products on the same platform is also quite different. For example, the geometric positioning accuracy of 756_1 TSX image is 7.104m, while the 986_1 TSX image is $381.923 \mathrm{~m}$. The traditional geometric calibration methods are usually based on single image for parameter calibration, and then use the parameter to correct other images under the same platform. However, in this experiment, parameter correction value for the same platform and different platform data products are not inherited, at this time the traditional calibration method is no longer applicable.
3.2.1 Hybrid Geometric Calibration: Based on this method, a hybrid geometric calibration experimental for 11 SAR images is carried out. Select the 630_4 image as the initial master image, and 5 GPS points are selected as GCPs. The TPs between the master and slave images are extracted from 630_4 image. Using these TPs to make geometric calibration of slave images, the calibrated slave images will be used as the next master image. By analogy, the two calibration parameters $\Delta t$ and $\Delta r$ of each image can be calibrated step by step. The transmission relationship between images is shown in Figure 4 . After the calibration of all the images is completed, the geometric accuracy of the calibrated SAR image using the indirect geometry positioning algorithm is shown in Table 3 . 


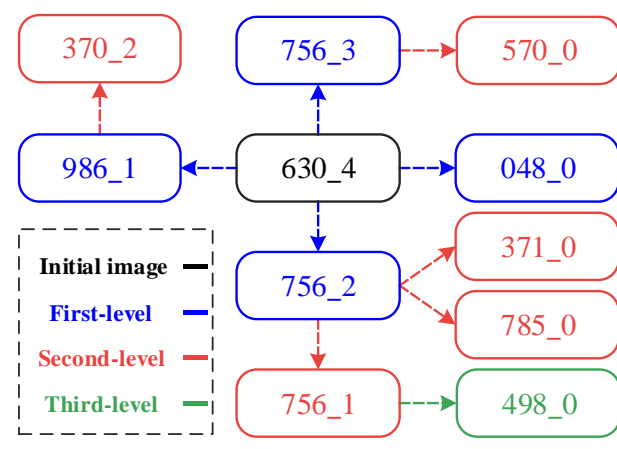

Figure 4. Transfer relation of images

\begin{tabular}{|c|c|c|c|c|c|c|}
\hline \multirow{2}{*}{ Image ID } & \multirow{2}{*}{$\begin{array}{c}\text { Number } \\
\text { of CPs }\end{array}$} & \multicolumn{2}{|c|}{ Correction Value } & \multicolumn{3}{|c|}{ Accuracy Evaluation After calibration } \\
\hline & & $\Delta t / \mathrm{ms}$ & $\Delta r / \mathrm{m}$ & Range/m & Azimuth/m & Plane $/ \mathrm{m}$ \\
\hline 630_4 & 11 & -0.435 & -3.527 & 0.958 & 1.908 & 2.135 \\
\hline 986_1 & 7 & 53.901 & -21.190 & 1.343 & 1.951 & 2.369 \\
\hline 756_3 & 5 & -65.919 & 71.004 & 1.159 & 2.280 & 2.558 \\
\hline 756_2 & 9 & -66.004 & 69.955 & 1.237 & 2.137 & 2.469 \\
\hline 370_2 & 7 & -0.444 & -6.792 & 1.370 & 2.148 & 2.548 \\
\hline 785_0 & 7 & -0.030 & -25.189 & 3.524 & 6.319 & 7.235 \\
\hline 570_0 & 5 & -17.582 & -20.827 & 2.673 & 5.479 & 6.096 \\
\hline 048_0 & 8 & -17.349 & -19.092 & 2.717 & 5.581 & 6.207 \\
\hline 371_0 & 11 & -16.069 & -20.954 & 4.371 & 4.091 & 5.987 \\
\hline 498_0 & 6 & -16.438 & -19.268 & 4.143 & 5.767 & 7.101 \\
\hline
\end{tabular}

Table 3. Accuracy evaluation of study images after the geometric calibration
Table 3 shows that the geometric positioning accuracy of TSX/TDX image is better than $3 \mathrm{~m}$ after geometric calibration, and the geometric positioning accuracy of GF-3 image is better than $7.5 \mathrm{~m}$. SAR image can achieve high-accuracy geometric positioning after calibration. In not considering the geometric calibration accuracy, the traditional geometric calibration method requires at least one GCP per scene image to complete the geometric calibration. However, the 11 image in paper only uses 5 GCPs to achieve a high-accuracy geometric positioning. The GCPs are concentrated in the same SAR image (630_4), to avoid a wide range of field measurement operation. As a whole, except for the start of the main image, no GCPs are used in the geometric calibration process of other images. This provides a way for SAR images to achieve wide-range, multi-platform geometric calibration without GCPs or sparse GCPs. In addition, the geometric positioning accuracy of the experimental data is quite different before the calibration, and the traditional geometric calibration method will no longer be applicable. This method can maximize the shielding effect of different satellite platforms, and has good versatility.

After geometric calibration, the geometric positioning accuracy of SAR images under different platforms still exists a certain gap, and the geometric positioning accuracy under the GF-3 platform is obviously lower than that of the TSX/TDX platform. The average value of geometric positioning accuracy of images under different platforms is calculated. The average geometric positioning accuracy of TSX, TDX, and GF-3 images is $2.460 \mathrm{~m}$, $2.525 \mathrm{~m}$, and $6.525 \mathrm{~m}$, respectively. The geometric positioning accuracy of TSX and TDX images is similar, and GF-3 image has a large gap compared to TSX/TDX images. There are two main reasons for this difference. First, the resolution of GF-3 images is lower than that of TSX/TDX images, which has influence on the accuracy of image pricking point and TPs matching. In general, higher resolution images are more conducive to high-accuracy geometric calibration; Secondly, as a sister star of TSX, TDX is similar in system parameters and imaging, and their geometric positioning accuracy is approximately equal. However, due to the relatively late start of SAR satellite in China, the geometric positioning accuracy of GF-3 satellite still has a certain gap compared with that of TSX/TDX. Although geometric calibration technology can greatly improve the geometric positioning accuracy of images, it is still limited by the performance of the platform itself.

Since geometric positioning accuracy of TSX/TDX images is not much different after geometric calibration, the effects of different levels of transfer on the geometric calibration results are analysed by combining the TSX/TDX images. According to the transfer relationship, two sets of data were selected. The first group selected images 630_4, 986_1 and 370_2, and their geometric positioning accuracy after geometric calibration was $2.135 \mathrm{~m}, 2.369 \mathrm{~m}, 2.548 \mathrm{~m}$, respectively. In the second group, images 630_4, 756_2, and 756_1 were selected in order, and the geometric positioning accuracy after geometric calibration was $2.135 \mathrm{~m}, 2.469 \mathrm{~m}$, and $2.877 \mathrm{~m}$, respectively. It can be seen that the geometric positioning accuracy of the image is successively reduced with the step-by-step transmission. This is because the geometric calibration of each level of image must have certain errors, and the process of level-by-level calibration makes the error propagate and accumulate. As a result, the geometric positioning accuracy of the image after the calibration is gradually reduced. Therefore, when the number of images is large, the transfer relationship between the images should be reasonably designed, and the transmission level should be as low as possible to ensure that the whole can achieve a good geometric calibration accuracy.

There are both plains and alpine lands in the experimental area. In order to analyse the effect of different elevations on the geometric calibration results, the geometric positioning accuracy of the three groups of calibrated images is statistically analysed. At the same time, considering that different levels of transmission have an impact on the geometric calibration accuracy, each group of images must be guaranteed to be at the same delivery level. In the first group, first-transfer TSX/TDX images 756_2 and 756_3 were selected, and the average elevations were $32.110 \mathrm{~m}$ and $448.793 \mathrm{~m}$, and the geometric positioning accuracy was $2.469 \mathrm{~m}$ and $2.558 \mathrm{~m}$, respectively; In the second group, the second-transfer GF-3 images 371_0, $570 \_0$ were selected, the average elevations were $32.089 \mathrm{~m}$, $489.643 \mathrm{~m}$, and the geometric positioning accuracy was $5.987 \mathrm{~m}$ and $6.096 \mathrm{~m}$, respectively; In the third group, second-transfer TSX/TDX images 756_1 and 370_2 were selected. The average elevations were $9.464 \mathrm{~m}$ and $282.103 \mathrm{~m}$, and the geometric positioning accuracy was $2.877 \mathrm{~m}$ and $2.548 \mathrm{~m}$, respectively. It can be seen that the average elevations in each set of data are relatively different, but the geometric positioning accuracy after calibration is relatively small, and the difference is less than $0.5 \mathrm{~m}$. For this paper, the effect of different terrain conditions on the geometric calibration results is mainly attributed to the difference in the elevation accuracy of the STRM under different terrain conditions. The higher the elevation accuracy of SRTM, the more accurate the geodetic coordinates of the TPs, 
and the corresponding geometric calibration accuracy will be improved. In general, the SRTM elevation accuracy under the alpine condition is lower than that in the plain. From Figure 3, it can be seen that the image TPs distributed in the relatively flat areas in the high mountain conditions, and distributed in steeper areas in the plain conditions, which offsets the elevation of SRTM under different terrain condition. Different terrain conditions have little effect on the geometric calibration results. The traditional geometric calibration method usually selects the corner reflector as the reference control target. The corner reflector has extremely strong backscattering energy and can be accurately identified. The recognition accuracy can generally reach sub-pixel level. The image coordinates of all GPS points in the experimental were obtained by artificial pricking. This process has pricking errors, which has a certain negative impact on the geometric calibration and accuracy evaluation of SAR images. In addition, the external DEM used in the experimental has a low resolution and has a certain influence on the geometric calibration (which will be discussed in 3.2.2), but it does not affect the comparison of the results of geometric calibration of multi-platform SAR images using the same DEM.

3.2.2 The Influence of External Data on Geometric Calibration Accuracy: The external DEM data needs to be used in the process of obtaining the geodetic coordinates of the TPs. The quality of the DEM determines the accuracy of the geodetic coordinates of the TPs, which will also affect the accuracy of subsequent geometric calibration. In order to ensure the generality of this method, DEM selects SRTM data with free, wide coverage and high-accuracy. If the DEM data with higher accuracy and higher resolution are used, the geometric calibration accuracy of this paper may be further improved. In order to study this problem, 3 high-resolution DEMs (TanDEM DEMs) were produced using TanDEM-X CoSSC data during the experimental process. The resolution was $12 \mathrm{~m}$, and the coverage range corresponded to images 756_2, 756_1, and 370_2, respectively. Using GPS points to evaluate the accuracy of the 3 TanDEM DEM and the corresponding area SRTM. By comparison, TanDEM DEM elevation accuracy (about $2 \mathrm{~m}$ ) is about 30\% higher than SRTM. TanDEM DEM is used as the external DEM data for geometric calibration, and the accuracy evaluation after the calibration is shown in Table 4.

\begin{tabular}{ccccc}
\hline \multirow{2}{*}{ Image ID } & \multirow{2}{*}{$\begin{array}{c}\text { Number } \\
\text { of CPs }\end{array}$} & \multicolumn{3}{c}{ Accuracy Evaluation After Calibration } \\
\cline { 3 - 5 } & 9 & Range/m & Azimuth/m & Plane/m \\
\hline $756 \_2$ & 1.348 & 1.524 & 2.035 \\
$756 \_1$ & 6 & 1.333 & 2.151 & 2.531 \\
$370 \_2$ & 7 & 1.458 & 1.943 & 2.429 \\
\hline
\end{tabular}

Table 4. Accuracy evaluation of study images after the geometric calibration

From Table 4, we can see that geometry calibration using the higher accuracy, higher resolution TanDEM DEM data, the geometric positioning accuracy of 756_2, 756_1, and 370_2 images respectively increased $0.434 \mathrm{~m}, 0.346 \mathrm{~m}$, and $0.119 \mathrm{~m}$. It is proved that high-accuracy and high-resolution DEM data can help to achieve higher geometric calibration accuracy.

On the other hand, this paper is based on 5 GCPs for geometric calibration of multi-platform SAR images. These 5 GCPs are basically distributed in four corner points $(\mathrm{N} 1 \sim \mathrm{N} 4)$ and center points (N5) of 630_4 image, and the location and ID of points are shown in Figure 3. The number of GCPs may have an impact on the geometric calibration accuracy, and this issue is being investigated. First, 1-5 GCPs are selected from 5 GCPs, and there are 31 combinations $\left(C_{5}^{1}+C_{5}^{2}+C_{5}^{3}+C_{5}^{4}+C_{5}^{5}\right)$. Then, the 630_4 image is calibrated using the GCPs after the combination. Finally, the geometric calibration accuracy was evaluated using $11 \mathrm{CPs}$, as shown in Figure 5.

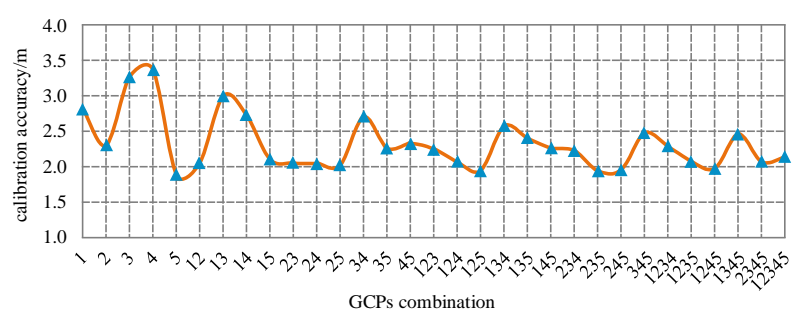

Figure 5. Accuracy evaluation of different GCP combinations

From Figure 5, we can see that when using N3 or N4 single GCP for geometric calibration, the accuracy of the calibration is low, and the subsequent geometric calibration accuracy is relatively low (such as 13,14, 34, 134, 345, 1345) are all associated with $\mathrm{N} 3$ or $\mathrm{N} 4$ point, indicating that the GCPs with low accuracy are not conducive to achieving high-accuracy geometric calibration. At the same time, it should also be noted that the geometric calibration accuracy under GCPs combination is better than using $\mathrm{N} 3$ or N4 GCP alone, indicating that when multiple GCPs are combined, highaccuracy GCPs will compensate for the low accuracy to a certain extent. When using 1-2 GCPs, the geometric calibration accuracy changes greatly, and the maximum accuracy difference can reach about $1.5 \mathrm{~m}$; When using 3-5 GCPs, the geometric calibration accuracy tends to be stable, and the accuracy difference can be controlled to about $0.5 \mathrm{~m}$. Therefore, it is recommended to use 3-5 GCPs for geometric calibration, by adding redundant observations to reduce the interference of low-accuracy GCP CPs, to ensure the stability and reliability of geometric calibration accuracy.

\section{CONCLUSION}

With the continuous launch of domestic SAR satellites, there is an urgent need to conduct in-depth research on the geometric calibration technology of spaceborne SAR, achieve highaccuracy geometric positioning of domestic satellite SAR images, and promote the practical application of domestic spaceborne SAR. In this paper, based on the R-D geometric positioning model, the influencing factors of geometric positioning accuracy are analysed, and a geometric calibration model is established. On this basis, a hybrid geometric calibration for multi-platform spaceborne SAR image with sparse GCPs method was proposed. Geometric calibration experiments were performed using 3 TerraSAR-X, 3 TanDEM$\mathrm{X}$, and 5 GF- 3 images. Using 82 GPSs for accuracy evaluation, the results show that the geometric positioning accuracy of TSX/TDX images after calibration is better than $3 \mathrm{~m}$, and the geometric positioning accuracy of GF-3 images is better than $7.5 \mathrm{~m}$, which improves the geometric positioning ability of multi-platform SAR images. It has reference value in actual data processing and application, especially for subsequent DOM or DEM integration of multi-scene.

In addition, there are still many problems to be studied in this paper, such as how to restrain and correct the propagation error in the process of step by step calibration; Due to the different left and right look-angle of satellite, there is a geometric distortion between the ascending and descending images, 
especially in mountainous areas, how to reduce the impact of distortion on the TPs matching. Subsequent research work will mainly start from these two aspects and provide more favourable support for hybrid geometric calibration study of this paper.

\section{ACKNOWLEDGEMENTS}

This work was supported by National Key R\&D Programme of China (NO: 2017YFB0502700), National Natural Science Foundation of China (NO: 41401529), Civilian Space Programme of China (DO: D010102), National Basic Surveying and Mapping Science and Technology Plan (No: 2018KJ0204/2018KJ0304). The authors would like to thank German Aerospace Center (DLR) for providing TerraSAR-X and TanDEM-X data (NO: CAL_VAL6993).

\section{REFERENCES}

Eineder M, Minet C, Steigenberger P, 2011a. Imaging Geodes Toward Centimeter-Level Ranging Accuracy With TerraSAR-X. IEEE Transactions on Geoscience and Remote Sensing, 49(2), pp. 661-671.

Cong Xiaoying, Balss U, Eineder M, 2012a. Imaging Geodesy Centimeter-Level Ranging Accuracy With TerraSAR-X: An Update. IEEE Geoscience and Remote Sensing Letters, 9(5), pp. 948-952.

Zhou Xiao, Zeng Qiming, Jiao Jian, 2014a. Analysis of TerraSAR-X Sensor Calibration Accuracy and Its Application. Remote Sensing Information, 29(2), pp. 31-35.

Schwerdt M, Brautigam B, Bachmann M, 2007. Efficient Calibration and First Results of TerraSAR-X. Proceedings of the Advanced SAR Workshop(ASAR).

Schwerdt M, Brautigam B, Bachmann M, 2010a. Final TerraSAR-X Calibration Results Based on Novel Efficient Methods. IEEE Transactions on Geoscience and Remote Sensing, 48(2), pp. 677-689.

Zhang Qingjun. 2017a, System Design and Key Technologies of the GF-3 Satellite. Acta Geodaetica et Cartographica Sinica, 46(3), pp. 269-277.

Ding Chibiao, Liu Jiayin, Lei Bin, 2017a. Preliminary Exploration of Systematic Geolocation Accuracy of GF-3 SAR Satellite System. Journal of Radars, 6(1), pp. 11-16.

Yang Shucheng, 2016b. Technique and Method on Synthetic Aperture Radar Stereoplotting. Wuhan: Wuhan University.

Curlander J C, Mcdonough R N, 1991. Synthetic Aperture Radar: Systems and Signal Processing. New York: Joho Wiley.

Cao Pengzhi, Gu Jianzheng, Xu Rongqing,1999a. Effect of Spaceborne SAR Attitude Errors and Yaw Steering on the Doppler Characteristics. Journal of Harbin Institute of Technology, 31(1), pp. 39-43.

Chen Erxue, 2004b. Study on Ortho-rectification Methodology of Spaceborne Synthetic Aperture Radar Imagery. Beijing: Chinese Academy of Forestry.
Guillaume Oller, Loic Rognant, Philippe Marthon, 2004a. Correlation and similarity measures for SAR image matching. International Society for Optics and Photonics, pp. 182-189.

Hanssen R. F., 2001a. Radar Interferometry Data Interpretation and Error Analysis. Journal of the Graduate School of the Chinese Academy of Sciences, 2(1), pp. V5-577-V5-580. 